\title{
Being tall compared to compared to being tall and being taller
}

\author{
Jaime Castillo-Gamboa, Alexis Wellwood, \& Deniz Rudin*
}

\begin{abstract}
This paper investigates the semantics of implicit comparatives (Alice is tall compared to Bob) and its connections to the semantics of explicit comparatives (Alice is taller than Bob) and sentences with adjectives in plain positive form (Alice is tall). We consider evidence from two experiments that tested judgments about these three kinds of sentence, and provide a semantics for implicit comparatives from the perspective of degree semantics.
\end{abstract}

Keywords. gradable adjectives; implicit comparatives; degree semantics; experimental semantics

1. Introduction. This paper investigates the semantics of implicit comparatives (1), and its connections to the semantics of explicit comparatives (2) and sentences with adjectives in their plain positive form (3): ${ }^{1}$

(1) Alice is tall compared to Bob.

(2) Alice is taller than Bob.

(3) Alice is tall.

We first present the results of two experiments probing the empirical profile of these three categories of sentence. We then leverage our results towards the development of a semantics for implicit comparatives that not only predicts the observed judgments, but interacts in the right ways with theories of explicit comparatives and unmodified positive form adjectives.

With respect to the relation between implicit and explicit comparatives, we investigate whether and how these two modes of comparison are distinct. Three relevant hypotheses are considered:

- Hypothesis 1: (1) and (2) are symmetrically entailing

- Hypothesis 2: (1) asymmetrically entails (2)

- Hypothesis 3: There is no relation between the acceptance of (1) and (2)

One could entertain the thought that (1) and (2) are simply different ways of saying the same thing. Such a thought would be falsified by evidence against Hypothesis 1. Evidence for Hypothesis 2 or 3 would be useful in fixing on the specific semantic differences between (1) and (2).

With respect to the relation between implicit comparatives and positive form adjectives, we investigate whether the evaluation of the plain positive form adjective is closely related to the evaluation of the implicit comparative. Specifically, we consider three hypotheses:

*We are grateful to George Zhiren Ye for his help in executing the experiments, and to Maribel Romero, Alexander Williams, Nurit Matuk-Blaustein, and reviewers for and attendees of ELM for their feedback. Authors: Jaime CastilloGamboa, University of Southern California (jcastillog@usc.edu), Alexis Wellwood, University of Southern California (wellwood@usc.edu) \& Deniz Rudin, University of Southern California (drudin@usc.edu).

${ }^{1}$ The distinction between implicit and explicit comparatives is explicitly formulated in Kennedy (2009). Pearson (2010) introduces a further distinction between 'strong' implicit comparatives, as in (1), and 'weak' implicit comparatives like Alice is taller compared to Bob. Our focus here is on strong implicit comparatives. Our ongoing experimental work examines weak implicit comparatives, however we leave discussion of such extensions to future work. 
- Hypothesis 4: (1) asymmetrically entails (3)

- Hypothesis 5: The acceptance of (1) is influenced by the acceptance of (3), despite lack of entailment

- Hypothesis 6: There is no relation between the acceptance of (1) and (3)

Hypotheses 4 and 5 are different ways of coding the idea that the computation of (1) is importantly related to that of (3), as we might understand from theories on which a phrase like compared to Bob modifies a positive form sentence while - er than Bob modifies the adjective. ${ }^{2}$ Evidence for Hypothesis 6, then, could provide indirect support for linking the semantics of implicit comparatives more closely with that of explicit comparatives.

We present our two experiments in $\S 2$. Both present participants with distributions of lines ranging in height along parameters that should affect the evaluation of plain positive form adjectives. Experiment 1 asks about tallness judgments for each line in each distribution using a sentence like (3), and Experiment 2 asks about comparative judgments for pairs of lines using sentences like (1) and (2). To preview our results, (i) we find evidence for an asymmetrical entailment from implicit comparatives to explicit comparatives, rejecting Hypotheses 1 and 3 and supporting Hypothesis 2, and (ii) we find no relation between the acceptance of implicit comparatives and plain positive form adjectives, rejecting Hypotheses 4 and 5 and supporting Hypothesis 6.

Buoyed by these results, in $\S 3$ we propose an account on which the semantics of implicit comparatives does not involve the computation of the plain positive form adjective, contra one way of understanding 'context-setting' views of implicit comparatives (Beck et al. 2004, Kennedy 2007). Importantly, we do not argue that our findings are incompatible with such views; however, we suggest that the difference-based degree semantics that we put forward (building on work by Solt 2009, Solt \& Gotzner 2012, Zhang \& Ling to appear) reflects those findings directly. To preview, (1) comes out as true just in case the difference between Alice and Bob's height is greater than the contextual standard for a difference-from-Bob's-height. \$4 summarizes, and highlights directions of ongoing work.

2. Experiments. We tested judgments about implicit comparatives, explicit comparatives and sentences in the positive form. Experiment 1 was designed to provide a baseline measure for the evaluation of plain positive form adjectives, and Experiment 2 was designed to probe (i) the relationship between the implicit and explicit comparatives (Hypotheses 1-3) and (ii) the relationship between the implicit comparative and adjectives in unmodified positive form (Hypotheses 4-6).

We recruited 60 unique participants on Amazon's Mechanical Turk who were each compensated \$2.00-\$2.40 for up to 12 minutes of their time. We restricted participation to Turkers located in the United States with a HIT approval rate of $99 \%$ or greater and a number of approved HITs at 1000 or greater. The studies were conducted in accordance with protocols approved by the University of Southern California's Institutional Review Board.

2.1. Methodology. We designed a set of 6 distributions of 12 thin rectangles which instantiated the cross of factors CLUSTERING (clust, unclust) and SCALING (flat, medium, steep) (see

\footnotetext{
${ }^{2}$ One example of a relation other than entailment between implicit comparatives and unmodified positive form adjectives (i.e., a pattern supporting Hypothesis 5, not Hypothesis 4) would be if implicit comparatives pragmatically implicate that the corresponding unmodified positive form sentence is false (Sawada 2009).
} 
Figure 1). We manipulated clustering to probe the impact of the availability of visually salient clusters on tallness judgments (Schmidt et al. 2009), and manipulated scaling as a proxy for just how visually salient those clusters are.

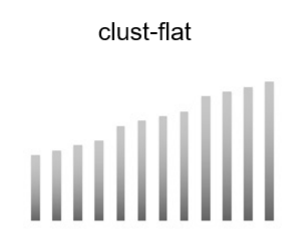

unclust-flat

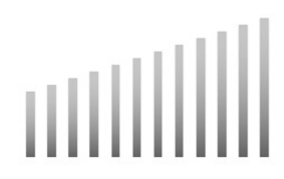

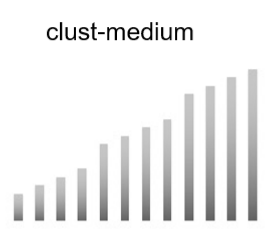

unclust-medium

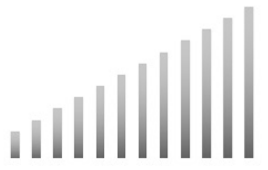

clust-steep

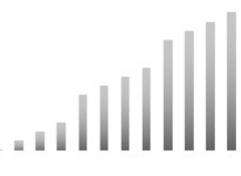

unclust-steep

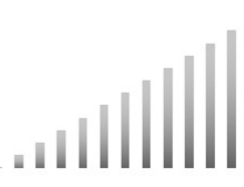

Figure 1: Distributions of lines used in Experiments 1 and 2

In Experiment $1(n=20)$, participants were asked to evaluate (4) against pictures of our distributions with, one at a time, each line in each distribution highlighted in green:

(4) The green line is tall.

In Experiment 2 ( $n=40)$, participants evaluated (5) and (6) (the factor SENTENCE, manipulated between subjects) by appropriate highlights of each possible combination of lines at a distance of 1, 3, and 5 lines apart (the factor DIFFERENCE) in each of our distributions:

(5) The blue line is tall compared to the red line.

(6) The blue line is taller than the red line.

Each of these combinations were tested both in a scenario where the blue line was taller than the red line and in one where the red line was taller than the blue line (the factor WINNER).

2.2. RESUlTS. Our statistical analyses involved logistic mixed effects regressions with random intercepts for subjects. We report $p$-values based on model comparisons between a maximal model $m$ and a model $m^{\prime}$ that subtracts a targeted variable. All analyses were conducted in $\mathrm{R}$ using RStudio and the lme4 package (Bates et al. 2014).

2.2.1. EXPERIMENT 1. We looked for signatures of judgments of tallness for individual lines (henceforth, 'tallness values'), which could be used as predictors for judgments about comparative tallness in Experiment 2. Potentially surprising given Schmidt et al. (2009) was the lack of effect of CLUSTERING on tallness values ( $p=0.76$; means: clust 0.38 , unclust 0.39 ). We did find an effect of SCALING $\left(\beta=3.16, \mathrm{SE}=1.25, \chi^{2}(1)=6.2, p=0.013\right)$, wherein (4) was accepted less as the distribution grew steeper (flat 0.44 , medium 0.38 , steep 0.33 ). These two factors did not interact $(p=0.24)$. The most significant effect in this dataset was that of the position of the green line in the distribution $\left(\beta=7.88, \mathrm{SE}=0.46, \chi^{2}(1)=1208.9, p<0.0001\right)$ (see Figure 2). 


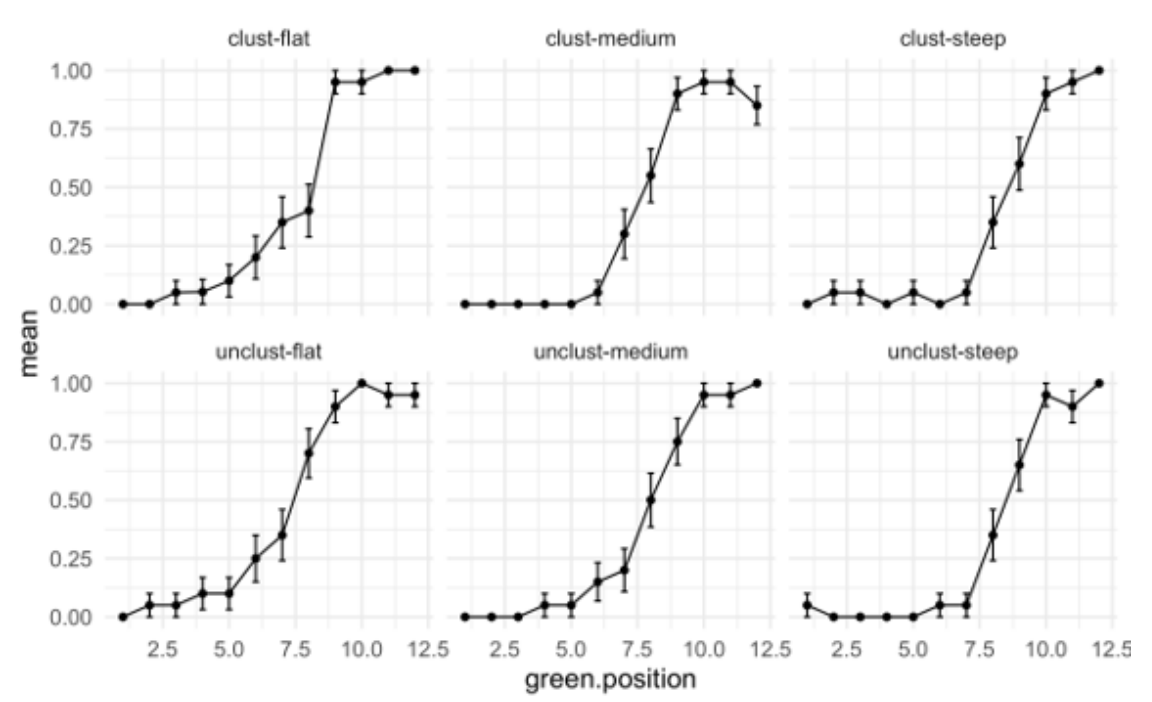

Figure 2: Proportion of tallness values in Experiment 1

We take from these results that the next experiment should look for (i) an effect of SCALING or (ii) some predictive power for the mean tallness values derivable from this dataset in the comparative judgment data. In order to constitute evidence for Hypotheses 4 or 5, we would have to observe any such effects selectively impacting the judgments for implicit comparatives.

2.2.2. EXPERIMENT 2. We looked for differences in the evaluation of implicit and explicit comparatives, and any relation between their evaluation and the patterns observed in Experiment 1 .

We expected that roughly half of the sentences would mark acceptance and half rejection. Thus, before conducting our analyses, we transformed raw 'yes' and 'no' responses into a measure called 'matching': a response matched just in case it marked acceptance of (5) or (6) when the blue line was the longer of the two, or it marked rejection and the red line was the longer.

We found an effect of WINNER $\left(\beta=-0.4, \mathrm{SE}=0.16, \chi^{2}(1)=6.44, p=0.011\right)$ corresponding to participants overall providing more matching responses in scenarios where the red line was the winner (mean matching values: Blue won 0.91, Red won 0.92). This effect was driven by the implicit comparatives, however: we observed an interaction effect between SENTENCE and WINNER $\left(\beta=1.37, \mathrm{SE}=0.31, \chi^{2}(1)=19.2, p<0.0001\right)$ showing a large difference in the same direction for implicit comparatives (Blue won 0.89, Red won 0.94), but a smaller difference in the opposite direction for explicit comparatives (Blue won 0.92, Red won 0.90) (see Figure 3).

We also found an effect of DIFFERENCE $\left(\beta=0.18, \mathrm{SE}=0.07, \chi^{2}(1)=6.79, p=0.009\right)$, such that responses overall matched more as the difference in position between the blue and red lines increased (means: 1-difference 0.91, 3- 0.92, 5- 0.93). Here, too, the effect was driven by the implicit comparative, as revealed in an interaction between SENTENCE and DIFFERENCE $\left(\beta=-0.32, \mathrm{SE}=0.14, \chi^{2}(1)=5.61, p=0.018\right)$ : matching responses increased for implicit comparatives along with the difference in position (1-difference $0.90,3-0.92,5$ - 0.94), while responses to the explicit comparatives did not (1-difference 0.91, 3- 0.91, 5- 0.91) (see Figure 4). 


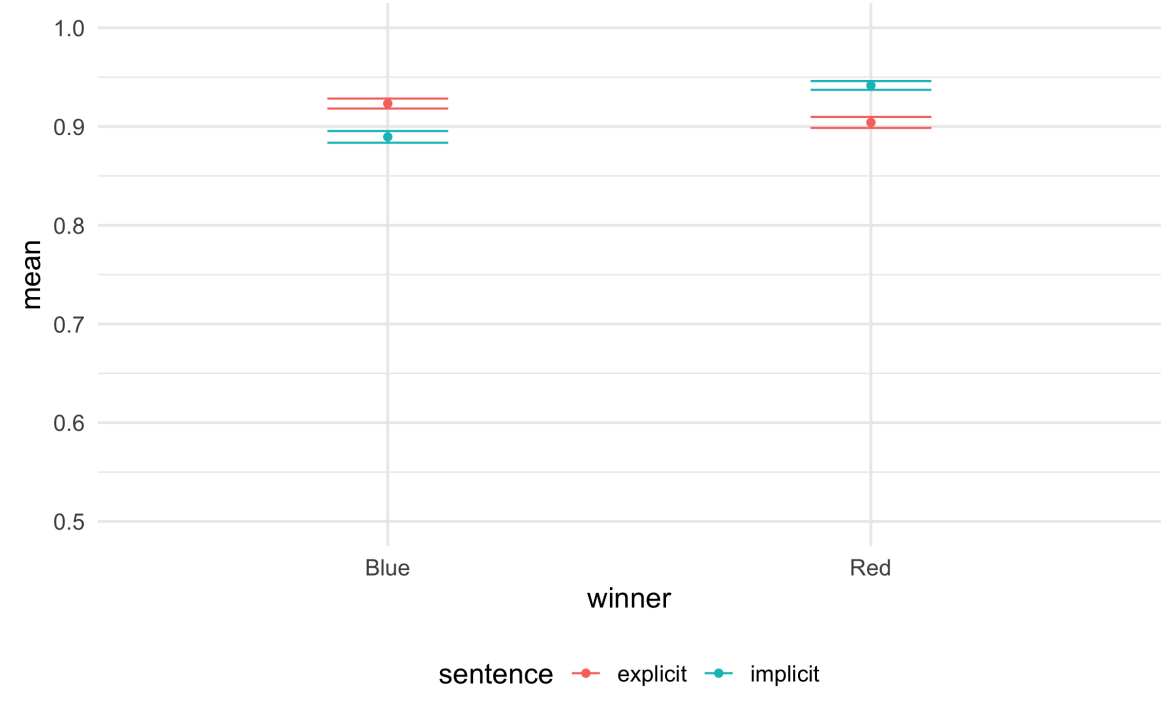

Figure 3: Effect of SENTENCE and WINNER on matching

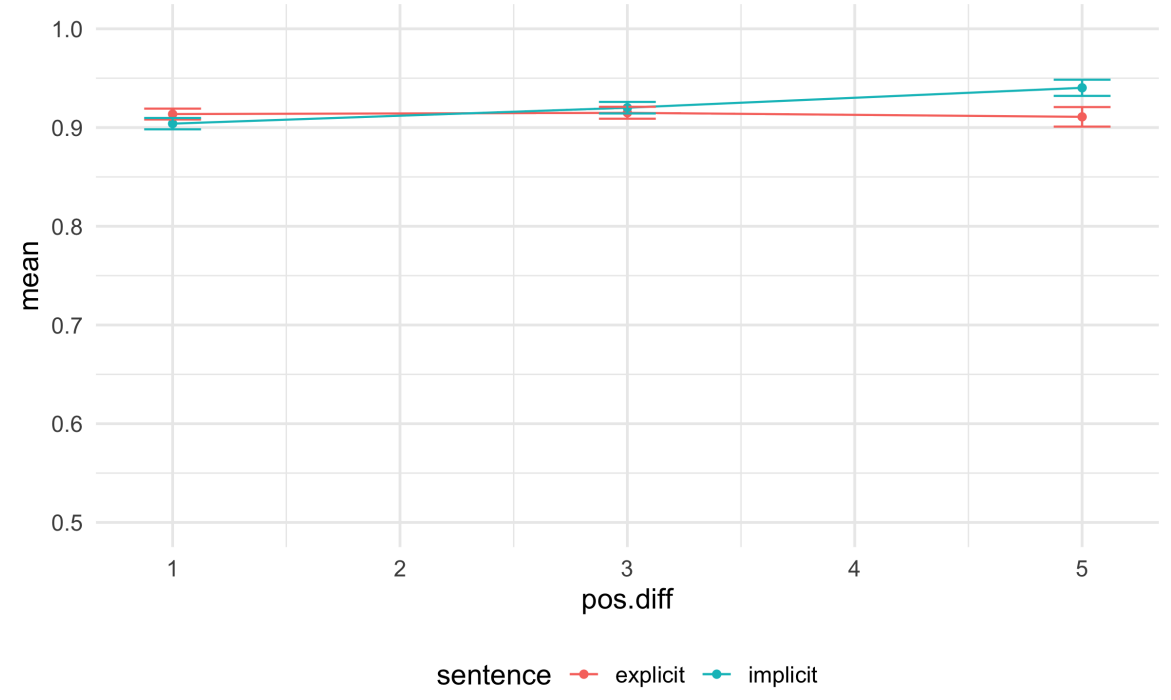

Figure 4: Effect of SENTENCE and DIFFERENCE on matching

What about potential signatures of tallness judgments on the evaluation of implicit and explicit comparatives? Crucially, SCALING did not play a predictive role here, neither overall $(p=0.58)$ nor in interaction with SENTENCE $(p=0.73)$. We didn't find any effects of tallness values on responses in Experiment 2, either. That is, we attempted analyses comparing SENTENCE, WINNER and the tallness values for those lines marked red and blue on each trial, and found no significant effects. There did not appear to be any predictive power for raw judgments of tallness on the evaluation of implicit comparatives, just as there wasn't for explicit comparatives. 
2.3. Discussion. The effects depicted in Figure 3 suggest that any positive difference between the heights of $y$ and $x$ is sufficient to make $x$ is tall compared to $y$ false. This provides evidence for the claim that an implicit comparative entails the corresponding explicit comparative. But it also suggests that not any positive difference between the heights of $x$ and $y$ is sufficient to make $x$ is tall compared to $y$ true. This provides evidence for the claim that an explicit comparative doesn't entail the corresponding implicit comparative. The effect depicted in Figure 4 suggests that, whereas any positive difference between the heights of $x$ and $y$ is enough to make the explicit comparative $x$ is taller than $y$ true, in order for the implicit comparative $x$ is tall compared to $y$ to be true, that difference must meet a certain threshold. Taken together, these findings allow us to reject Hypotheses 1 and 3 from $\S 1$, and lend support to Hypothesis 2.

The lack of any observed alignment between judgments of tallness and comparative judgments supports the plain intuition that explicit comparatives $x$ is taller than $y$ neither entail nor are entailed by their positive correspondents $x$ is tall, and furthermore suggests the same for implicit comparatives: $x$ is tall compared to $y$ neither entails nor is entailed by $x$ is tall. These findings provide support for Hypothesis 6 and against Hypotheses 4 and 5 from $\S 1$.

Any positive difference between the heights of $x$ and $y$ is sufficient to make the explicit comparative $x$ is taller than $y$ true, but the likelihood of our participants judging that $x$ is tall compared to $y$ increased as the difference between $x$ and $y$ increased (see Figure 4). Because this pattern is gradient, not categorical, it suggests the existence of 'borderline cases' of implicit comparatives that are not obviously true and not obviously false.

3. Analysis. We can now provide a semantics for implicit comparatives that captures the main findings from our experiments. We first introduce some background assumptions in $\$ 3.1$ about the semantics of explicit comparatives and plain positive form adjectives. In $\$ 3.2$, we present our preferred account of implicit comparatives, according to which their semantics involves the interaction between the morpheme POS and what we call 'difference measure functions', which result from the interaction between compared to-phrases and gradable adjectives. Finally, in $\S 3.3$, we show that this account correctly predicts support for Hypothesis 2 and Hypothesis 6, and the existence of borderline cases for implicit comparatives.

3.1. BACKGROUND ON DEGREE SEMANTICS. We assume a semantics for gradable adjectives according to which they denote measure functions that map objects to degrees (Cresswell 1976, Stechow 1984, Kennedy 1999, 2007). ${ }^{3}$ For instance, the adjective tall denotes the function tall, which maps objects onto their heights:

$$
\llbracket \operatorname{tall} \rrbracket=\lambda x \cdot \operatorname{tall}(x)
$$

On this approach, gradable adjectives combine with degree morphology to produce properties of individuals. This is precisely what happens in the case of explicit comparatives. In (2), tall combines with -er and the than-clause to produce a property that is instantiated by an object just in case its height is greater than Bob's height:

\footnotetext{
${ }^{3}$ The main alternative to degree semantics is delineation semantics (Klein 1980, Doetjes et al. 2011, Burnett 2017). From this perspective, gradable adjectives denote functions from objects to truth-values relative to delineations, which represent different ways of classifying objects from a given domain. For discussion of implicit comparatives in the context of delineation semantics, see Rooij (2011a, 2011b) and Kennedy (2011, 2019).
} 
(8) $\llbracket$ taller than Bob $\rrbracket=\lambda x \cdot \operatorname{tall}(x)>\operatorname{tall}(b)$

Explicit comparatives are then given truth conditions as in (9):

(9) $\llbracket$ Alice is taller than $\operatorname{Bob} \rrbracket=1$ iff $\operatorname{tall}(a)>\operatorname{tall}(b)$

In general, an explicit comparative $x$ is more $G$ than $y$ is true just in case $x$ 's degree of $g$-ness is greater than $y$ 's degree of $g$-ness, where $g$ is the measure function denoted by $G$.

The interpretation of the positive form is less straightforward in the context of degree semantics. Since, unlike other adjectives, gradable adjectives do not map individuals onto truth-values, they cannot combine directly with the subject of the sentence. To overcome this difficulty, proponents of degree semantic approaches often maintain that, in the positive form, gradable adjectives occur along with an unpronounced morpheme POS, which denotes a function from measure functions to properties of individuals. For instance, in (3), POS combines with tall and returns a property that is instantiated by an object just in case its height is equal to or greater than a certain standard:

$$
\llbracket \text { tall POS } \rrbracket=\lambda x \cdot \operatorname{tall}(x) \geq s t
$$

The value of $s t$ is determined by a standard function $\mathbf{s}$ that maps measure functions onto degrees:

$$
\llbracket \mathrm{POS} \rrbracket=\lambda g \cdot \lambda x \cdot g(x) \geq \mathbf{s}(g)
$$

The truth conditions of (3) are then as follows:

$$
\llbracket \text { Alice is tall POS } \rrbracket=1 \text { iff } \operatorname{tall}(a) \geq \mathbf{s}(\text { tall })
$$

Two features of sentences in the positive form are relevant for our purposes. On the one hand, they are context-sensitive. For instance, (3) might be true relative to a context where the relevant domain includes only Alice's friends from work and false relative to a context where the relevant domain includes only the members of Alice's basketball team. On the other hand, they are vague. Consider, for instance, the context involving the members of Alice's basketball team. Relative to that context, it seems impossible to draw a sharp line between those individuals who are tall and those who are not. Consequently, some individuals are borderline cases of tall. ${ }^{4}$

There are different ways of accounting for these features. Here we follow Kennedy (2007) and assume that the explanation for both of them is to be found in the semantics of POS. Let's start with context-sensitivity. On Kennedy's view, POS determines different standard functions relative to different contexts:

$$
\llbracket \mathrm{POS} \rrbracket^{c}=\lambda g \cdot \lambda x \cdot g(x) \geq \mathbf{s}_{c}(g)
$$

These standard functions determine different standard degrees, which explains the context-sensitivity of sentences like (3):

$$
\begin{aligned}
& \llbracket \text { tall POs } \rrbracket^{c}=\lambda x \cdot \operatorname{tall}(x) \geq \mathbf{s}_{c}(\text { tall }) \\
& \llbracket \text { Alice is tall POs } \rrbracket^{c}=1 \text { iff } \operatorname{tall}(a) \geq \mathbf{s}_{c}(\text { tall })
\end{aligned}
$$

\footnotetext{
${ }^{4}$ This is just an intuitive way of highlighting two features of the positive form and is intended to be compatible with theories that treat vagueness as a form of context-sensitivity, as in Fara (2000) and Raffman (1996).
} 
The vagueness of the positive form is explained by the way in which standard functions select a standard degree. Given a context $c, \mathbf{s}_{c}$ determines a standard "in such a way as to ensure that the objects that the positive form is true of 'stand out' in [c], relative to the kind of measurement that the adjective encodes" (Kennedy 2007:207). If we were to draw a sharp line between tall and non-tall objects, we would violate the 'standing out' requirement.

3.2. A DIFFERENCE-BASED SEMANTICS FOR IMPLICIT COMPARATIVES. Following Fults (2005, 2006:\$2.3), we assume that compared to-phrases enter the compositional process and combine directly with the gradable adjective. This contrasts with views that treat compared to-phrases as pragmatic context setters, such as those in Beck et al. (2004) and Kennedy (2007). ${ }^{5}$ In this section, we introduce a difference-based account of implicit comparatives, building on prior work by Solt (2009:§4.5), Solt \& Gotzner (2012), and Zhang \& Ling (to appear). In section 3.3, we show that it accounts for the main results discussed in section 2 .

According to the difference-based view, an implicit comparative $x$ is G compared to $y$ is true relative to a context $c$ just in case the difference in $g$-ness between $x$ and $y$ is greater than or equal to a contextually determined standard, which is selected in such a way that the objects that count as $G$ compared to $y$ in $c$ stand out with respect to the difference between their $g$-ness and $y$ 's $g$-ness. For instance, (1) [Alice is tall compared to Bob] is true relative to $c$ just in case the difference in height between Alice and Bob is greater than or equal to a certain contextually determined standard, which is selected in such a way that the objects that count as tall compared to Bob in $c$ stand out with respect to the difference between their height and Bob's height.

In order to obtain these truth conditions compositionally, we proceed as follows. First, compared to-phrases denote functions from measure functions to what we'll call difference measure functions ('DMFs', for short). Given a measure function $g$ that maps an object onto its degree of $g$-ness, the DMF of $g$ with respect to $x$ maps an object onto the difference between its degree of $g$-ness and $x$ 's degree of $g$-ness. For instance, compared to Bob denotes a function that takes a measure function $g$ and returns the DMF of $g$ with respect to Bob, i.e., a function that maps an object onto the difference between its degree of $g$-ness and Bob's degree of $g$-ness:

$$
\llbracket \text { compared to Bob } \rrbracket=\lambda g \cdot g^{-b} \text {, where } g^{-b} \text { abbreviates } \lambda x \cdot g(x)-g(b)
$$

On this picture, compared to-phrases are the result of combining the compared to-phrase with noun phrases such as Bob. This leaves us with the following lexical entry for compared to:

$$
\llbracket \text { compared to } \rrbracket=\lambda x \cdot \lambda g \cdot g^{-x}
$$

The next step is to combine compared to Bob with tall. By that process, we obtain the DMF in (18), which maps an object onto the difference between its height and Bob's height:

$$
\llbracket \text { tall compared to } \mathrm{Bob} \rrbracket=\lambda x \cdot \text { tall }^{-b}(x)
$$

\footnotetext{
${ }^{5}$ On these views, the function of compared to-phrases is to fix the context in which the embedded sentence is to be evaluated. Thus, an implicit comparative $x$ is $G$ compared to $y$ is true relative to a context $c$ just in case $x$ is $G$ is true relative to a different context $c^{*}$ that bears a certain relation to $c$. According to Kennedy (2007), $c^{*}$ is just like $c$ except that the only individuals in the domain are those being compared, i.e., $x$ and $y$. On the other hand, Beck et al. (2004) suggest that $c^{*}$ is a context just like $c$ except that the value that the standard function corresponding to $c^{*}$ assigns to the measure function denoted by $G$ is $y$ 's degree of $G$-ness.
} 
As with other measure functions, the DMF in (18) combines with POS and returns a property of individuals, which is instantiated by an object just in case the difference between that object's height and Bob's height is greater than or equal to the standard assigned to the DMF by the standard function in $c$ :

$$
\llbracket \text { tall compared to Bob POS } \rrbracket=\lambda x \cdot \text { tall }^{-b}(x) \geq \mathbf{s}_{c}\left(\text { tall }^{-b}\right)
$$

Finally, the truth-conditions of (1) are given in (20).

$$
\llbracket \text { Alice is tall compared to Bob POs } \rrbracket^{c}=1 \text { iff } \text { tall }^{-b}(a) \geq \mathbf{s}_{c}\left(\text { tall }^{-b}\right)
$$

That is, Alice is tall compared to Bob relative to a context $c$ just in case the difference in height between Alice and Bob is greater than or equal to the standard assigned by the standard function in $c$ to the DMF of tall with respect to Bob.

3.3. SOME FEATURES OF THE DIFFERENCE-BASED VIEW. Here we show that the differencebased view can account for three important features of implicit comparatives, which were suggested by the results from our experiments: (i) $x$ is tall compared to $y$ entails but is not entailed by $x$ is taller than $y$ (Hypothesis 2), (ii) there is no relation between the acceptance of $x$ is tall compared to $y$ and $x$ is tall (Hypothesis 6), and (iii) $x$ is tall compared to $y$, just like $x$ is tall, has borderline cases.

3.3.1. Being tall compared to COMPARED TO being taller. Recall that, on our semantics, $x$ is taller than $y$ is true just in case $\operatorname{tall}(x)>\operatorname{tall}(y)$, i.e. just in case $\operatorname{tall}(x)-\operatorname{tall}(y)$ is greater than 0 . Now, according to the difference-based view, $x$ is tall compared to $y$ is true just in case $\operatorname{tall}(x)-\operatorname{tall}(y)$ is greater than or equal to the contextually determined standard for the DMF tall $^{-y}$. Given the 'standing out' requirement imposed by the semantics of POS, it is plausible to assume that no context has 0 as the standard of $\operatorname{tall}^{-y}$. Thus, $\operatorname{tall}(x)-\operatorname{tall}(y)$ being greater than or equal to the standard amounts to $\operatorname{tall}(x)-\operatorname{tall}(y)$ being greater than 0 . That is, to $x$ is taller than $y$ being true. Therefore, an implicit comparative entails the corresponding explicit comparative. The converse, however, is not true. The fact that $\operatorname{tall}(x)-\operatorname{tall}(y)$ is greater than 0 doesn't entail that it is greater than or equal to the contextually determined standard for $\operatorname{tall}^{-y}$. For $\operatorname{tall}(x)-\operatorname{tall}(y)$ might be a number between 0 and said standard. Therefore, an explicit comparative doesn't entail the corresponding implicit comparative. In sum, the difference-based view predicts Hypothesis 2.

3.3.2. Being tall compared to COMPARED TO being tall. Given a context $c, x$ is tall is true just in case $x$ 's height is greater than or equal to $c$ 's standard for tall, whereas $x$ is tall compared to $y$ is true just in case tall $(x)-\operatorname{tall}(y)$ is greater than or equal to $c$ 's standard for $\operatorname{tall}^{-y}$. The following cases show that our semantics correctly predicts $x$ is tall compared to $y$ to neither entail nor be entailed by $x$ is tall:

- Suppose Alice is $160 \mathrm{~cm}$ tall and Bob is $150 \mathrm{~cm}$ tall, and consider a context $c$ where the standard for tall is $170 \mathrm{~cm}$ and the standard for tall ${ }^{-y}$ is $5 \mathrm{~cm}$. Relative to $c$, the interpretation of (1) is true, but that of (3) is false.

- Suppose Carol is $180 \mathrm{~cm}$ tall and Bob is $179 \mathrm{~cm}$ tall and consider a context $c$ where the standard for tall is $170 \mathrm{~cm}$ and the standard for tall $^{-y}$ is $5 \mathrm{~cm}$. Relative to $c$, the interpretation of Carol is tall compared to Dan is false, but that of Carol is tall is true. 
3.3.3. Being tall compared to AND POS. Our experiments did not find a connection between the evaluation of implicit comparatives and that of plain positive form adjectives. However, implicit comparatives are analytically similar to such occurrences of adjectives in that both involve the interaction between POS and measure functions. Whereas in $x$ is tall, POS combines with the measure function tall, in $x$ is tall compared to $y$, it combines with the DMF tall $^{-y}$. As a result, we expect implicit comparatives to be both vague and context sensitive:

- Given a context $c, \mathbf{s}_{c}$ maps tall ${ }^{-y}$ onto a standard degree in such a way that those who count as tall compared to $y$ stand out in $c$ in virtue of their difference in height with $y$. As a result, (i) it is not be possible to draw a sharp line between those who count as tall compared to $y$ and those who don't, and (ii) there are borderline cases of tall compared to $y$.

- The morpheme POS determines different standard functions relative to different contexts. When these functions are applied to tall ${ }^{-y}$, different degrees are determined as standard degrees. This makes implicit comparatives context-sensitive.

The vagueness of implicit comparative was suggested at the end of $\$ 2.3$. Moreover, although our experiments didn't provide evidence for the context-sensitivity of implicit comparatives, there are cases that give us reason to think that they are in fact context-sensitive. Suppose Sadie and Fido are two Golden Retrievers. Sadie is $80 \mathrm{~cm}$ tall and Fido is $78 \mathrm{~cm}$ tall. Relative to a context where the relevant domain includes all sorts of dogs, (21) might be considered false:

(21) Sadie is tall compared to Fido.

However, relative to a context where the relevant domain includes only Golden Retrievers participating in a very prestigious dog show, (21) might be considered true. It is plausible to explain this phenomenon by maintaining that different contexts provide different standards for DMFs.

4. Conclusion. In this paper, we have provided a difference-based semantics for implicit comparatives supported by and reflective of the results from two formal judgment experiments. On our approach, implicit comparatives involve some of the same degree-theoretic compositional elements as sentences with unmodified positive form adjectives, i.e., the morpheme POS and an expression denoting a measure function. This helps to explain some of the similarities between the two kinds of sentence, as well as the differences between implicit and explicit comparatives. At the same time, the fact that expressions of the form tall compared to $y$ denote DMFs helps to account for the entailment from $x$ is tall compared to $y$ to $x$ is taller than $y$.

As we noted above, we understand a difference-based account to more directly capture the empirical data uncovered by our experiments than alternative, 'context-setter' views. However, we do not understand our data or discussion to rule out such views. In ongoing work, we are testing the evaluation of the same three kinds of sentence as investigated here with different contextual manipulations (including a no-context variant). We are also investigating the semantics of weak implicit comparatives (see note 1) and its interactions with the semantics of strong implicit comparatives. We anticipate that the results of our subsequent studies will shed additional light on the evaluation of competing approaches to the semantics of comparison. 


\section{References}

Bates, Douglas, Martin Maechler, Benjamin M. Bolker \& Steven Walker. 2014. Ime4: Linear mixed-effects models using Eigen and S4. R package version 1.1-7. http://CRAN.Rproject.org/package=lme4.

Beck, Sigrid, Toshiko Oda \& Koji Sugisaki. 2004. Parametric variation in the semantics of comparison: Japanese and english. Journal of East Asian Linguistics 13. 289-344. https://doi.org/10.1007/s10831-004-1289-0.

Burnett, Heather. 2017. Gradability in natural language. Oxford: Oxford University Press. https://doi.org/10.1093/acprof:oso/9780198724797.001.0001.

Cresswell, Maxwell John. 1976. The semantics of degree. In Barbara Partee (ed.), Montague grammar, 261-292. New York, NY: Academic Press. https://doi.org/10.1016/B978-0-12-5458504.50015-7.

Doetjes, Jenny, Katerina Soucková \& Camelia Constantinescu. 2011. A neo-Kleinian approach to comparatives. In Ed Cormany, Satoshi Ito \& David Lutz (eds.), Proceedings of Semantics and Linguistic Theory (SALT), 19, 124-141.

Fara, Delia Graff. 2000. Shifting sands: An interest-relative theory of vagueness. Philosophical Topics 28(1). 45-81. https://doi.org/10.5840/philtopics20002816.

Fults, Scott. 2005. Comparison and compositionality. In John Alderete, Chung hye Han \& Alexei Kochetov (eds.), Proceedings of the 24th West Coast Conference on Formal Linguistics, 146154. Simon Fraser University.

Fults, Scott. 2006. The structure of comparison. College Park, MD: University of Maryland dissertation.

Kennedy, Christopher. 1999. Projecting the adjective. New York: Routledge. https://doi.org/10.4324/9780203055458.

Kennedy, Christopher. 2007. Vagueness and grammar. Linguistics and Philosophy 30. 1-45. https://doi.org/10.1007/s10988-006-9008-0.

Kennedy, Christopher. 2009. Modes of comparison. In Malcolm Elliott, James Kirby, Osamu Sawada, Eleni Staraki \& Suwon Yoon (eds.), Papers from the 43rd Annual Meeting of the Chicago Linguistic Society. Volume I: The Main Session, 139-163. Chicago: Chicago Linguistic Society.

Kennedy, Christopher. 2011. Vagueness and comparison. In Paul Égré \& Nathan Klinedinst (eds.), Vagueness and language use, 73-97. Basingstoke: Palgrave Macmillan. https://doi.org/10.1057/9780230299313.

Kennedy, Christopher. 2019. The sorites paradox in linguistics. In Sergi Oms \& Elia Zardini (eds.), The sorites paradox, 246-262. Cambridge: Cambridge University Press. https://doi.org/10.1017/9781316683064.

Klein, Ewan. 1980. A semantics for positive and comparative adjectives. Linguistics and Philosophy 4. 1-45. https://doi.org/10.1007/BF00351812.

Pearson, Hazel. 2010. How to do comparison in a language without degrees: A semantics for the comparative in Fijian. In Martin Prinzhorn, Viola Schmitt \& Sarah Zobel (eds.), Proceedings of Sinn Und Bedeutung, 14, 356-372.

Raffman, Diana. 1996. Vagueness and context relativity. Philosophical Studies 81. 175-192. 
Rooij, Robert van. 2011a. Implicit versus explicit comparatives. In Paul Égré \& Nathan Klinedinst (eds.), Vagueness and language use, 51-72. Basingstoke: Palgrave Macmillan. https://doi.org/10.1057/9780230299313.

Rooij, Robert van. 2011b. Vagueness and linguistics. In Giuseppina Ronzitti (ed.), Vagueness: A guide, 123-170. Dordrecht: Springer. https://doi.org/10.1007/978-94-007-0375-9.

Sawada, Osamu. 2009. Pragmatic aspects of implicit comparison: An economy-based approach. Journal of Pragmatics 41(6). 1079-1103. https://doi.org/10.1016/j.pragma.2008.12.004.

Schmidt, Lauren, Noah Goodman, David Barner \& Joshua Tenenbaum. 2009. How tall is tall? Compositionality, statistics, and gradable adjectives. In Niels Taatgen \& Hedderik van Rijn (eds.), Proceedings of CogSci 31,3151-3156.

Solt, Stephanie. 2009. The semantics of adjectives of quantity. New York, NY: City University of New York dissertation.

Solt, Stephanie \& Nicole Gotzner. 2012. Experimenting with degree. In Anca Chereches (ed.), Proceedings of SALT 22, 166-187.

Stechow, Arnim von. 1984. Comparing semantic theories of comparison. Journal of Semantics 3(1-2). 1-77. https://doi.org/10.1093/jos/3.1-2.1.

Zhang, Linmin \& Jia Ling. to appear. The semantics of comparatives: A difference-based approach. Journal of Semantics . 